\title{
Factors Associated with the Caregivers' Desire to Institutionalize Persons with Dementia: A Cross-Sectional Study
}

\author{
Sophie Vandepitte ${ }^{a} \quad K$ oen Putman ${ }^{b}$ Nele Van Den Noortgate ${ }^{c}$ \\ Sofie Verhaeghe ${ }^{a}$ Eric Mormont ${ }^{d,}$ e Lisa Van Wilder ${ }^{a}$ \\ Delphine De Smedt ${ }^{a}$ Lieven Annemans ${ }^{a}$ \\ ${ }^{a}$ Department of Public Health and Primary Care, Faculty of Medicine and Health Sciences,

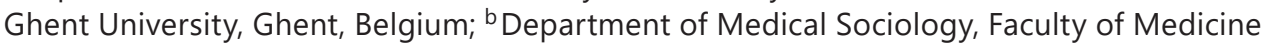

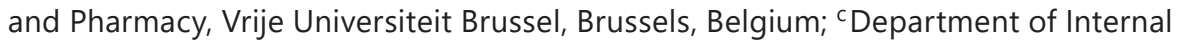 \\ Medicine, Faculty of Medicine and Health Sciences, Ghent University, Ghent, Belgium; \\ ${ }^{d}$ CHU UCL Namur, Service de Neurologie, Yvoir, Belgium; 'Université Catholique de Louvain, \\ Institute of NeuroScience, Louvain-la-Neuve, Belgium
}

\section{Keywords}

Institutionalization · Informal care · Alzheimer

\begin{abstract}
Background/Aims: Dementia is one of the main reasons for institutionalization among the elderly. Few studies have explored factors associated with the caregivers' (CG) desire to institutionalize (DTI) a person with dementia (PWD). The objective of this study is to identify modifiable and non-modifiable psychosocial and sociodemographic factors associated with a caregiver's DTI. Methods: Cross-sectional data of 355 informal CG of community-dwelling PWD were analyzed. Several characteristics were identified in CG and PWD to be included in a multivariable regression model based on the purposeful selection method. Results: Positively modifiable associated factors were: higher CG burden, being affected by behavioral problems, and respite care use. Positively associated non-modifiable factors were: CG older age, being professionally active, and CG higher educational level. Cohabitation and change of professional situation were negatively associated. Conclusion: Although no causality can be assumed, several practical recommendations can be suggested. First of all, these results reconfirm the importance of multicomponent strategies, especially support aimed at decreasing burden and in learning coping strategies. Also, CG might benefit from information about support options, such as respite care services. Finally, special attention should be given to older and working CG. In the latter, flexible and adaptive working conditions might alleviate burden and therefore reduce the DTI of the PWD.


Vandepitte et al.: Factors Associated with the Caregivers' Desire to Institutionalize Persons with Dementia

\section{Introduction}

Dementia is one of the main reasons for institutionalization among the elderly [1]. Previous research indicated that, on average, almost half (43\%) of the persons with dementia live in a residential care setting, a proportion that further rises when the disease evolves to more advanced stages [2]. To date, most studies have investigated predictors or factors associated with the informal caregivers' (CG) final decision to institutionalize the person with dementia (PWD) [3]. However, few studies have explored factors associated with the CG desire to institutionalize (DTI) the PWD [4, 5].

The DTI scale of Morycz $[6,7]$ has proven to be well predictive of actual institutionalization $[4,5,7,8]$. Earlier research concluded that CG who had a DTI a cognitively impaired elder person were 1.5 times more likely to actually institutionalize them within a period of six to nine months $(p<0.001)$ than those without a desire [8]. DTI is thus a useful evaluation tool in predicting institutionalization in interventions with short follow-up. Otherwise, identifying the risk factors of dementia CG' DTI allows to develop effective strategies to delay institutionalization. The latter is preferable because it positively affects the health-related quality of life of the PWD (Vandepitte et al. [9]) [10], and avoids rapid declines in health and eventually increased mortality [11]. Even more, staying at home is suspected to have financial benefits for the healthcare system as a result of avoided institutionalizations $[12,13]$.

The few studies focusing on factors associated with DTI already indicated that DTI is influenced by several characteristics of CG and PWD. Spruytte et al. [8] concluded, based on their sample of 144 CG of cognitively impaired elderly that, not living together, the presence of behavioral problems, and not being religious were significant predictors of developing a DTI. Spitznagel et al. [5] concluded that CG with a DTI experienced a higher burden, had better dementia knowledge, poorer family relationships, and less social support compared to those without DTI ( $n=72)$. In accordance, Gallagher et al. [4] concluded that caregiver burden and caregiver depression were significant predictors of the DTI ( $n=84$, Alzheimer patients). Another study revealed that being a male caregiver, not being the spouse, and a higher use of formal services were predictors of developing a preference for institutional care [14]. Finally, a recent trial $(n=171)$ revealed some counterintuitive results. The study concluded that being a housewife primary caregiver was positively associated with DTI, and being a female PWD was negatively associated with DTI [15].

Current evidence shows that clear insights into the underlying associated factors of a DTI remain very restricted due to the small sample sizes of these trials, the limited number of potential associated factors under investigation, the latter often in combination with the lack of a properly built statistical model allowing to understand the complex nature of these factors, and the lack of an exclusive focus on dementia. Evidence regarding the predictors of DTI should be increased. Therefore, the objective of this study is to identify the modifiable and non-modifiable psychosocial and sociodemographic factors associated with a CG DTI.

\section{Design and Methods}

\section{Participants}

The baseline data from an ongoing prospective study titled "Effectiveness and cost-effectiveness of an in-home respite care program in supporting informal CG of people with dementia" were used, see Vandepitte et al. [16]. Ethical approval (B670201526906) for this trial was obtained, and the trial is registered on clinicaltrials.gov, ID: NCT02630446.

A total of 355 informal CG of community-dwelling persons diagnosed with dementia participated in the study. They were recruited from 6 different memory clinics, a geriatric daycare clinic, several Alzheimer cafés (monthly meeting place for peers), local info points and expert centers for dementia, the Flemish Alzheimer 
Vandepitte et al.: Factors Associated with the Caregivers' Desire to Institutionalize

Persons with Dementia

Table 1. Desire to institutionalize scale modified (McCaskill et al. [7]): scale items

1 In the past 6 months, have you considered a nursing home, boarding home, or Yes (=1) or no (=0) assisted living for the care-recipient (CR)?

2 In the past 6 months, have you felt that the CR would be better off in a nursing Yes (=1) or no $(=0)$ home, boarding home or assisted living?

3 In the past 6 months, have you discussed the possibility of a nursing home, Yes (=1) or no (=0) boarding home or assisted living with family members or others?

\begin{tabular}{lll}
\hline 4 & In the past 6 months, have you discussed the possibility with CR? & Yes $(=1)$ or no $(=0)$ \\
\hline 5 & In the past 6 months, have you taken any steps toward placement? & Yes $(=1)$ or no $(=0)$ \\
\hline 6 & $\begin{array}{l}\text { In the past } 6 \text { months, are you likely to move the CR to another living } \\
\text { arrangement? }\end{array}$ & Yes $(=1)$ or no $(=0)$
\end{tabular}

league, a Belgian in-home respite care service, a sickness fund, an independent home healthcare service, and 16 general practitioners. All sites were located in Belgium. In each setting, eligible participants were identified by the health care professionals, the study was described, and informal CG were asked if they were willing to participate. When they gave their verbal consent, contact information was forwarded to the research team who then contacted them by phone. During this call a home visit was planned to sign the informed consent and complete the baseline assessment.

To be eligible for the study, the CG had to provide informal care, they had to identify themselves as the main person responsible for the informal care (primary caregiver), and fluently understand Dutch or French. The care recipient had to be formally diagnosed with dementia (Alzheimer disease, vascular dementia, frontotemporal dementia, and lewy body dementia) and reside in the community. Exclusion criteria were CG with severe cognitive impairment or psychiatric comorbidity, and dementia patients living in a residential setting.

\section{Measures}

To identify candidate variables to enter the multivariable statistical model, the literature on factors associated with DTI was reviewed. The following variables were included in this study:

\section{Caregiver DTI}

The Desire-to-Institutionalize scale, developed by Morycz et al. [6], is widely known as a reliable predictor for future institutionalization [7, 17]. In this study, a modified version was used [7]. Because no valid translation existed for Belgium, a forward-back translation was undertaken [16]. The scale contains six questions (displayed in Table 1) evaluating the CG DTI the PWD in the last six months by answering either "yes" or "no." The higher the total score (maximum score $=6$ ), the greater the desire [16]. DTI was recoded into a dichotomous variable according to previous research $[4,5]$. In this way, 2 groups were identified: CG without desire (score on the DTI $=0$ ) and CG with a DTI (DTI $\geq 1$ ).

\section{Caregiver Burden}

Burden was measured using the Zarit Burden Interview, a 22-item validated self-report questionnaire developed to examine subjective burden of CG of PWD. The scale uses a 5 point Likert scale, ranging from "never" to "nearly always present." The final score ranges between 0 (no burden) and 88 (high burden) [18].

Caregiver Health Related Quality of Life and Perceived Health

Health-related quality of life and self-perceived health was measured using the EQ-5D-5L, which is a standardized non-disease specific value-based instrument to describe and value self-perceived health status [19]. The instrument consists of 5 health-related domains (mobility, self-care, daily activities, pain, and depression/anxiety). Each domain has 5 answer categories: no problems, slight problems, moderate problems, severe problems, and extreme problems resulting in a 5 digit health profile. The profile can be translated into a utility value ranging between -0.285 and 1 with the use of a published algorithm [20]. Additionally, the instrument contains a Visual Analog Scale (VAS) to measure one's health today, ranging from 0 to 100 in which " 0 " means the worst imaginable health while " 100 " represents the best imaginable health. 
Resource Use of the PWD

Resource use of the PWD was collected using the internationally validated Resource Use in Dementia instrument [21]. Some small adjustments were made in agreement with the Belgian context and with the setting of this study. Data used in the current study were: being hospitalized, emergency room care in the last 6 months, outpatient visits in the last month, use of homecare in the last month, and use of respite care services in the last 6 months. The 5 different types of resource use were recoded into 5 dichotomous variables (use versus nonuse).

Time Spent in Caregiving

Time spent in caregiving was also measured using a part of the Resource Use in Dementia questionnaire [21]. In this questionnaire, caregiver time is evaluated by asking how much time is spent on activities of daily living (ADL), instrumental ADL, and supervision on an average caregiving day in the past 30 days. According to common practice [22], the total reported time was capped at maximum $18 \mathrm{~h}$ per day to avoid overestimation [23].

Dementia Severity

Severity of dementia was measured during an interview with the caregiver using the Global Deterioration Scale (GDS) [23], which is a valid and reliable instrument that distinguishes 7 stages of dementia based on cognitive decline. Because patients in this study were all diagnosed with dementia, only stage 4 until stage 7 could occur, namely: moderate cognitive decline (stage 4), moderately severe cognitive decline (stage 5), severe cognitive decline (stage 6), and very severe cognitive decline (stage 7).

Level of Dependency of the PWD

ADL of the PWD was measured using a Belgian adapted version of the Katz Index of Independence in ADL [24]. This instrument measures a person's ability to perform ADL based on a total score on six functions: bathing, dressing, toileting, transferring, continence, and feeding. Additionally, potential disorientation in time and place was also asked in this questionnaire. Scores range from 8 to 32.

Behavioral Problems of the PWD

To measure the frequency of problematic behaviors in the PWD and the reaction of CG to these problems, the Revised Memory and Behavior Problems Checklist was used [25]. This validated caregiver self-report measure contains 24 items including 3 domains (depression, memory-related problems, and disruptive behaviors) comprising 2 scales. One scale measures the frequency of problem behaviors of the PWD (ranging from "never occurs" to "occurs daily or more often") and the other measures the reactions of the caregiver to these problematic behaviors (ranging from "not at all" to "extremely"). Only the mean score of the first scale (sum of 8 disruptive behavior items divided by 8) was used in this study (range 0-4), because behavioral disturbances are especially known to be emotionally challenging for CG.

\section{Sociodemographics}

The following sociodemographic variables were selected for inclusion in the model based on existing literature: age, gender, educational level and civil state of the dyads, professional status of the caregiver and changes in this status due to caregiving, and cohabitation and type of relationship within the dyads. Educational level was divided into 3 groups according to the International Standard Classification of Education [26]: low educated (from early childhood education to lower secondary education), medium educated (upper secondary education to post-secondary non-tertiary education), and high educated (short-cycle tertiary education to doctoral degree or equivalent). Civil state comprised 2 groups: living alone or living together. Professional status was dived into: active on the labor market, currently inactive (e.g., sick leave, time credit), or being retired. Relationship between the dyads contained the following categories: spouse/partner, child, or other family member/friend. Cohabitation was dichotomous: one could either score yes or no. Concerning changes in professional status, the caregiver was asked if he/she had changed his/her working situation - in terms of working less hours - to better deal with caregiving tasks. Here, CG could also score yes or no.

\section{Statistical Analyses}

First, descriptive statistics of the participant characteristics were undertaken to describe the study sample $(n=355)$. For continuous variables, mean and standard deviations are shown, for categorical variables, percentages are used. 
Vandepitte et al.: Factors Associated with the Caregivers' Desire to Institutionalize

Persons with Dementia

Next, the purposeful selection strategy for logistic regression analysis, described by Bursac et al. [27], was applied to generate the "best" model. DTI was identified as dependent variable. According to this strategy, univariable logistic regressions were performed on the selected candidate variables to explore unadjusted associations. An arbitrary cut-off point of $p<0.25$ based on the Wald test was used to evaluate appropriateness for inclusion in the multivariable model $[27,28]$. Eligible variables were then simultaneously included as candidates in a multivariable model. Next, an iterative process of variable selection was performed, each step excluding the candidate with the highest $p$ value. This process was repeated until only associated factors at the arbitrary level of $p<0.15$ remained in the model $[27,28]$. Additionally, each time a certain candidate was removed in the iterative process, differences between $-2 \log$ likelihood estimates of the former and new model were checked to evaluate whether deletion was the correct decision [28]. If differences between both were considerable, the candidate should not be excluded because it may not be associated with DTI by itself, but influence associations of others with DTI [29]. In the last step, variables that were initially not eligible for inclusion in the multivariable model are now simultaneously added. The same iterative process was then repeated (read: only for the new added variables) at the corresponding arbitrary cut-off $p$ value of 0.1 . The latter was helpful to identify variables, not related to DTI by themselves, but having an important contribution in combination with other variables [27].

Collinearity diagnostics (Variance Inflation Factor and Tolerance) were checked. Quality of the final model was evaluated based on: Nagelkerke R Square (to evaluate the total variance explained in the dependent variable by the model), and the Hosmer and Lemeshow Test of the goodness of fit (to evaluate if the model is a good fit to the data). Overfitting of the model was prevented by checking the following equation: number of cases $\geq 10 *$ number of parameters included in the model [30].

In the final model, a type I error level of $\alpha=0.05$ was taken into account. Regression coefficients, ORs, and Wald estimates were interpreted to evaluate the magnitude and direction of the association. Independent associated factors based on the model are represented as modifiable or non-modifiable associated factors in the results section. All analyses were conducted using IBM SPSS statistical software version 24.0.

\section{Results}

\section{Sample Description}

A response rate of $77 \%$ was reached in this study, implying that, of the 465 CG recruited in the participating settings 355 eventually completed the baseline evaluation during a home visit after they were contacted by the researchers.

\section{Characteristics of the Study Participants}

The descriptive statistics of the dyads characteristics are outlined in Table 2. The mean age of the $355 \mathrm{CG}$ was 67.4 years $(\mathrm{SD}=12.04)$. About two-third of CG were women $(65.4 \%)$. When evaluating their professional situation, $20 \%$ were active on the labor market (formally employed), more than two-third were retired (67.9\%), and $12.1 \%$ were non-active due to several reasons (e.g., sick leave, time credits). To better deal with their caregiver role, $11.8 \%$ of them had changed their professional situation by reducing their working hours. Almost half of the CG were highly educated (42.8\%), while $27.9 \%$ were low educated. About $52.4 \%$ of the CG lived in Flanders, 38.6\% in Wallonia, and 9\% in Brussels. The majority of CG lived together with the PWD (79.7\%). The type of relationship within the dyads was mostly spouse or partner $(66.5 \%), 26.2 \%$ was a son or daughter of the dementia person, and the remaining CG were friends or other family members $(7.3 \%)$. The mean age of the persons with dementia was 78.7 (SD = 8.62). Slightly more than half of the PWD were women $(53.5 \%)$. About $70.7 \%$ of them still had a partner or spouse. About $44.2 \%$ of the PWD were low educated and $29.2 \%$ were highly educated.

About two-third of the CG had a DTI the dementia patient (63.4\%). CG scored on average 33.03 (SD = 16.18) on the Zarit Burden Scale. CG' health-related quality of life, measured by the EQ-5D-5L, was on average $0.74(\mathrm{SD}=0.22)$. Their perceived health, measured by the VAS 
Table 2. Descriptive statistics and bivariate analyses

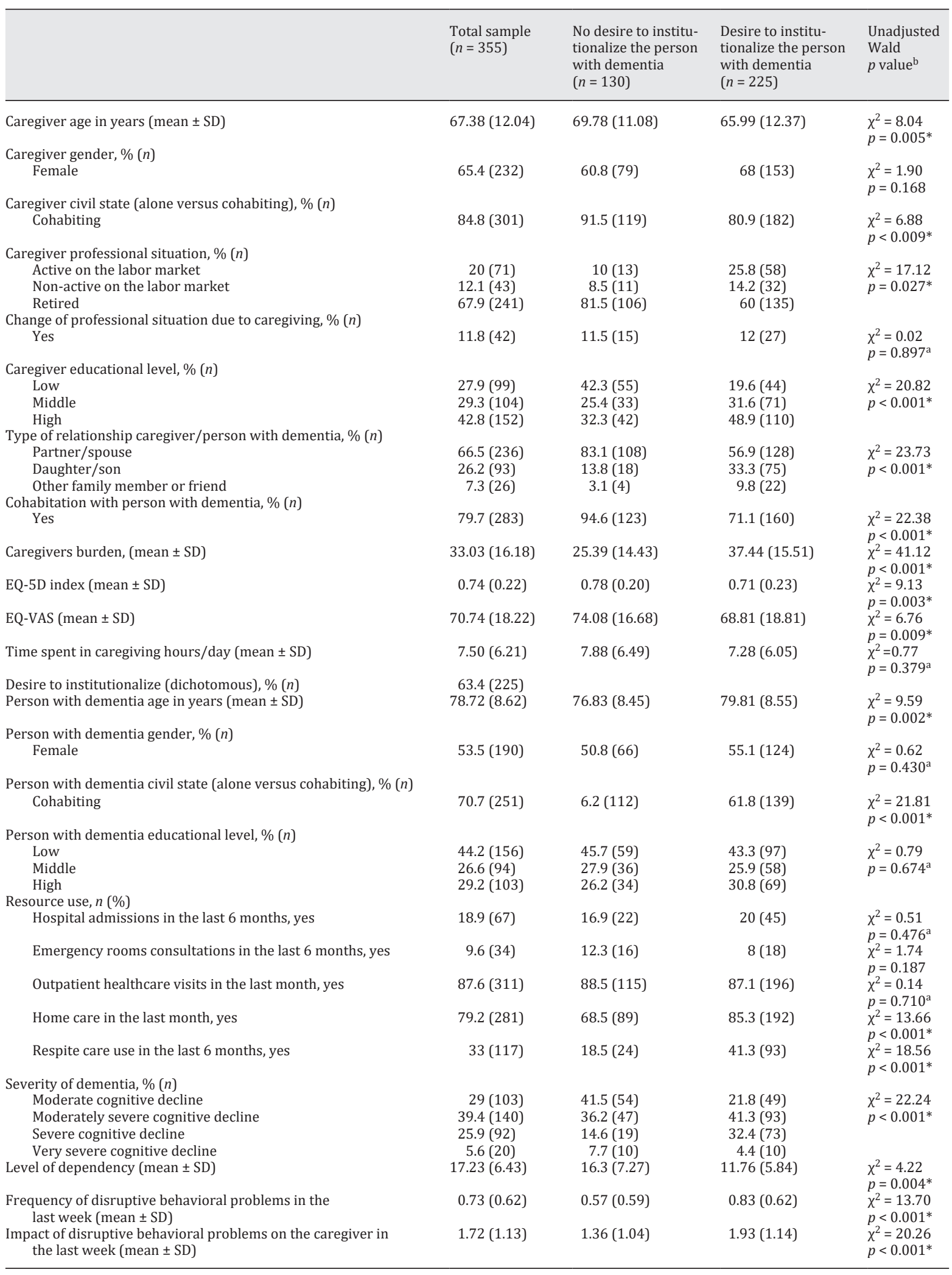

* Significant difference at $p=0.05$ level. ${ }^{a}$ Excluded as candidate for multivariable logistic regression model based on arbitrary level of $p=0.25$ according to the purposeful selection method described by Bursac et al. [27]. ${ }^{\mathrm{b}}$ Based on the univariable logistic regressions to evaluate eligibility for the multivariable model. 
Vandepitte et al.: Factors Associated with the Caregivers' Desire to Institutionalize Persons with Dementia

Table 3. Results from the multivariable logistic regression model based on purposeful selection (Bursac et al. [27])

\begin{tabular}{|c|c|c|c|c|}
\hline & B & OR $(95 \% \mathrm{CI})$ & Wald & $p$ value \\
\hline Age of caregiver & 0.042 & $1.043(1.00-1.088)$ & 3.862 & $0.049 *$ \\
\hline Age of person with dementia & 0.029 & $1.029(0.995-1.065)$ & 2.769 & 0.096 \\
\hline Cohabitation $^{\mathrm{a}}$ & -2.532 & $0.080(0.028-0.223)$ & 23.068 & $0.000^{*}$ \\
\hline Professional situation & & & 6.036 & $0.049 *$ \\
\hline Professional situation ${ }^{\mathrm{b}}$ & 0.162 & $1.176(0.370-3.741)$ & 0.075 & 0.784 \\
\hline Professional situation ${ }^{\mathrm{c}}$ & -1.213 & $0.297(0.091-0.973)$ & 4.022 & $0.045^{*}$ \\
\hline Change of professional situation & -1.122 & $0.325(0.117-0.904)$ & 4.640 & $0.031 *$ \\
\hline Educational level of caregiver & & & 13.987 & $0.001 *$ \\
\hline Educational level of caregiverd & 1.358 & $3.887(1.861-8.121)$ & 13.046 & $0.000 *$ \\
\hline Educational level of caregiver ${ }^{\mathrm{e}}$ & 0.979 & $2.662(1.338-5.297)$ & 7.783 & $0,005^{*}$ \\
\hline Outpatient visits in the last month ${ }^{\mathrm{f}}$ & -0.761 & $0.467(0.198-1.104)$ & 3.011 & 0.083 \\
\hline Emergency room consult in the last 6 months $^{f}$ & -0.856 & $0.425(0.164-1.097)$ & 3.127 & 0.077 \\
\hline Respite care in the last 6 months $^{\mathrm{f}}$ & 1.314 & $3.721(1.953-7.088)$ & 15.97 & $0.000^{*}$ \\
\hline Self-perceived burden & 0.051 & $1.052(1.029-1.076)$ & 20.099 & $0.000^{*}$ \\
\hline Impact of disruptive behaviors on caregiver & 0.334 & $1.396(1.031-1.890)$ & 4.653 & $0.031^{*}$ \\
\hline
\end{tabular}

* Significant difference at 0.05 level. ${ }^{a}$ Cohabitation yes/no. ${ }^{\mathrm{b}}$ Active yes/no. ${ }^{\mathrm{c}}$ Retired yes/no. ${ }^{\mathrm{d}}$ Medium

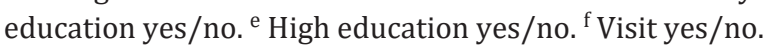

scale (Visual Analog Scale), was on average 70.74 (SD = 18.22). About $18.9 \%$ of the PWD were hospitalized in the last 6 months, while $9.6 \%$ had visited the emergency room in the same time period. In the last month, $87.6 \%$ had an outpatient visit, and $79.2 \%$ used home care. Finally, $33 \%$ used respite care services in the last six months. Concerning dementia severity, $29 \%$ had moderate cognitive decline (GDS 4), 39.4\% had moderately severe cognitive decline (GDS 5), $25.9 \%$ had severe cognitive decline (GDS 6), and 5.6\% had very severe cognitive decline (GDS 7). CG spent on average 7.5 ( $\mathrm{SD}=6.21$ ) hours per day on caregiving. At patient level, the mean level of dependency was $17.23(\mathrm{SD}=6.43)$. They scored on average $0.73(\mathrm{SD}=0.62)$ on the disruptive behaviors frequency subscale and $1.72(\mathrm{SD}=1.13)$ on the disruptive behaviors reaction subscale of the Revised Memory and Behavioral Problems Checklist.

\section{Associated Factors of a DTI}

Unadjusted associated factors are outlined in Table 2, adjusted associated factors, as a result of the multivariable regression model, are represented in Table 3.

CG with a DTI were more often highly educated $(p<0.001)$, had a higher burden $(p<$ $0.001)$, were more affected by the behavioral disruptive behaviors of the PWD $(p<0.001)$, and used more respite care services in the last six months $(p<0.001)$. CG without DTI were more often: cohabiting $(p=0.009)$ or living together with the PWD $(p<0.001)$, spouse/ partner of the PWD $(p<0.001)$, retired ( $p=0.027)$, had a higher health-related quality of life on the EQ-5D index ( $p=0.003)$, and on the EQ-VAS ( $p=0.009)$.

Looking at the PWD, DTI was positively associated with a higher age $(p=0.002)$, frequent occurrence of disruptive behaviors $(p<0001)$, severe dementia $(p<0.001)$, home care use $(p<0.001)$, and being physically dependent $(p=0.004)$. Finally, the PWD of CG without DTI were more often cohabiting ( $p<0.01$ ) with someone (not necessarily the caregiver).

Modifiable Independent Associated Factors in the Multivariate Analysis

Burden (OR 1.052; 95\% CI 1.029-1.076; $p<0.001$ ) was independently positively associated with DTI, meaning that the odds of having a DTI increased when burden increased ( $5 \%$ 
Vandepitte et al.: Factors Associated with the Caregivers' Desire to Institutionalize Persons with Dementia

per extra point on the Zarit Burden Scale). Even more, based on the Wald estimate, burden was the most important independent associated factor in the multivariable model $\left(\chi^{2}=20.1\right.$, $p<0.001$ ). Impact of disruptive behavioral problems on the caregiver (OR 1.396; 95\% CI 1.031-1.890; $p=0.031$ ) was also positively associated with DTI. Finally, the resource use variable "use of respite care" (OR 3.721; 95\% CI 1.953-7.088; $p<0.001$ ) was independently and positively associated with DTI (odds of DTI was 3.7 times higher in users compared to non-users).

Non-Modifiable Independent Associated Factors in the Multivariate Analysis

The most important non-modifiable independent associated factor was cohabitation $\left(\chi^{2}=23.07\right.$, OR 0.08; 95\% CI 0.028-0.223; $\left.p<0.001\right)$. When caregiver and patient lived together, the odds of having a DTI decreased (with 92\%). Professional situation and change in professional situation to better deal with caregiving duties, were also negatively associated with DTI, that is: when CG were retired (OR 0.297; 95\% CI 0.091-0.973; $p=0.045$ ) or changed their professional status (OR 0.325; 95\% CI 0.117-0.904, $p=0.031$ ), the odds of having DTI decreased (respectively with 70 and 67\%). On the contrary, DTI was associated with high educated CG (OR 2.662; 95\% CI 1.338-5.297; $p=0.005$ ) and with increasing caregiver age (OR 1.043; 95\% CI 1.00-1.088; $p=0.049$ ).

Nagelkerke R square pointed out that $46 \%$ of the total variance in the dependent variable DTI was explained by the model. The Hosmer and Lemeshow goodness-of-fit-test indicated a good fit of the model $\left(\chi^{2}=3.46, p=0.9\right)$.

\section{Discussion}

The findings of this study confirm that several modifiable and non-modifiable psychosocial and sociodemographic factors are associated with the desire of CG to institutionalize the PWD. Even more, a multifactorial association of several factors was demonstrated by the multivariable regression model and indicates that developing a certain desire is a difficult process.

Before fully discussing the results, several limitations and strengths of this research should be mentioned allowing to better understand the context of these findings. A first limitation is the cross-sectional nature of the investigated data. Because the data were captured at one point in time, no causality could be assumed and therefore only associations and no predictions could be made. Second, recruitment of the dyads was not at random and was carried out by several organizations often specialized in dementia treatment or informal caregiving support. Although not intended nor necessary for the study objectives, it is important to mention that this convenience sample is not representative for the entire dementia caregiver population. For example, highly educated CG may be overrepresented. Although unambiguous (inter)national numbers of sociodemographic dementia caregiver characteristics are lacking, a recent report of the Flemish government indicated that in general $25.4 \%$ of informal CG (not dementia-specific) was highly educated [31]. This number is lower than the percentage of highly educated CG in this study (42.8\%). Consequently, CG in complete denial, not accepting any help or support, and probably most in need, are likely to be not present in this study. Also, because the ongoing study evaluates the effectiveness of an in-home support program to support informal CG of community-dwelling dementia patients, included CG may be more inclined to keep the dementia patient at home. Besides these limitations, important strengths of this study can be addressed as well. First of all, the sample size of this study is substantially larger than in previous studies focusing on DTI $[4,5,8,14,15]$. Second, the focus is strictly on informal CG of PWD while others often only included them as a subsample $[8,32]$. This strict focus is preferable, because earlier research indicated that 
caring for someone affected by dementia is more burdensome than caring for someone who is not cognitively impaired. This is caused by the difficulties CG often experience in managing behavioral problems and the constant need for supervision [33,34]. A third strength is the use of internationally validated tools, as well as the wide range of potentially influencing factors under investigation. A final strength was the use of a properly built multivariable regression model indicating good quality after evaluation. In this study, the purposeful selection method described by Bursac et al. [27] was chosen because it has several advantages, especially when researchers are interested in associations and not merely predictions. These advantages can be addressed to its algorithm, able to better preserve confounders in addition to significant covariates. As a result, a high variance of DTI was indeed explained by the regression model. In similar studies, a substantially lower percentage could only be explained [8] or total variance explained was not even reported [4, 5].

Self-reported burden was identified as the most important associated factor, in accordance with previous work investigating predictors and factors influencing DTI $[4,5]$ and actual placement $[11-13,35]$. These results highlight the importance of effective caregiver support to decrease their burden [16,36]. Another modifiable independent associated factor was the impact of behavioral disturbances on the CG. More specifically, being more affected by disruptive behaviors was positively associated with having a DTI. This finding corresponds to earlier evidence indicating that self-efficacy protects against developing a DTI [4]. On the contrary, the frequency of these behaviors was not independently associated with DTI. The fact that the frequency of these problems as such is less important than the emotional impact they can cause, also corresponds to previous work $[5,14]$. Coping strategies can be learned when behavioral problems in dementia cannot be treated [36,37]. Additionally, it would be interesting to further investigate the causal relationship between the presence of behavioral symptoms and the DTI, in particular the role of DTI in the development or aggravation of behavioral symptoms in the PWD. To our knowledge, this was the first study investigating the association between DTI and the change of professional situation to better deal with caregiving tasks. The fact that our analyses indicate that this better adapted working situation protects against DTI is very interesting for policy makers. In the light of their preference for community-based healthcare and the expected decrease of available CG due to demographical changes, policymakers could consider to elaborate more flexible work schedules and conditions for this group allowing them to maintain both roles. Further longitudinal research could demonstrate the causal relationship between the working conditions for CG and their DTI. Use of respite care in the last six months was also positively associated with DTI in the final model. At first sight, this is a rather counterintuitive result, however, this seemingly paradoxical observation was also found in several other studies. López et al. [14] concluded that the use of formal services was associated with DTI, while others found a positive association between actual institutionalization and service use [11], use of community-based services $[12,13]$, and respite care in general [13]. It is important to keep in mind that an association does not assume any causal relationship and that this perceived paradox can be attributed to various reasons. First of all, respite care is often used as a "rehearsal" before actual institutionalization or when a crisis occurs while waiting for institutionalization [13, 14]. Second, CG often wait too long to accept help and when they finally do, respite care becomes a transition towards institutionalization [38]. Third, when CG start using services, they become aware of the possibilities [11], barriers are overcome, and they accept that they cannot do everything by themselves. Before, they did not even consider institutionalization, now they learn to accept the possibility when it becomes necessary. A comprehensive (qualitative) study of unmet caregiver needs would be interesting to develop more insights in this matter $[14,39]$. Finally, health-related quality of life and perceived health was not independently associated with DTI in accordance with previous work [14,32]. 
Vandepitte et al.: Factors Associated with the Caregivers' Desire to Institutionalize Persons with Dementia

Concerning non-modifiable factors, corresponding to previous work, it was concluded that caregiver age [11, 12, 35], professional situation [40], and educational level [12] were positively associated with DTI. The latter can be explained by the fact that highly educated CG may be better informed about the possibilities, may have less problems to pay for institutional care, and may be less prepared to adapt or give up their professional career for caregiving. On the contrary, cohabitation protects against DTI. This finding is in accordance with other studies $[8,12]$ and suggests that living together has a more important influence on DTI than the type of relationship within the dyads, which was not independently associated. Indeed, it is to be expected that more time, organization, and effort is needed to provide informal care when not sharing the same household. Also, the relationship is probably more intense between people living together and separation by institutionalization may have a greater impact on the CG' life. Finally, in contrary to previous work, caregiver spousal status and gender was not associated with DTI in our model. This could be explained by the demographical chances in our society: more women are active on the labor market and therefore feel less obligated to stay home and care for someone, as well as the changed family structures also play a role.

This research has revealed interesting associations and indicated a multifactorial association of several modifiable and non-modifiable factors on DTI. These identified associated factors can be used by policy makers to elaborate future directions in community-based dementia care. First of all, the independent association of several caregiver and patient characteristics with DTI reconfirms the need for multicomponent strategies. Especially, support aimed at decreasing burden and in learning to better cope with behavioral disturbances of the PWD are certainly worth the effort. Also, CG need to be well informed about the support they can receive as well as the resource use available for community-dwelling dementia patients. Important here is that this information should be given in time and in a structured and easy accessible way. Finally, identification of non-modifiable factors was important as well, because they can help detect CG at risk or in extra need for help. In this light, special attention should be given to older CG and CG active on the labor market. Future longitudinal research should investigate if introducing flexible and adaptive working conditions, allowing them to maintain their role as caregiver and employee, has an effect on the care of people with dementia at home. Additionally, it would be valuable to investigate other plausible causal relationships between the identified characteristics in both dyad members associated with DTI. In this way, more precise recommendations could be made, and carefully selected interventions known to be effective in reducing risk factors of DTI could be implemented.

\section{Acknowledgments}

The authors would like to thank the collaborating physicians, organizations, job students, volunteers, and informal CG for their valuable contribution in this study.

\section{Disclosure Statement}

We have no conflict of interest to declare.

\section{Funding Source}

This work was supported by the Baillet Latour fund (E/01447/15). 
Vandepitte et al.: Factors Associated with the Caregivers' Desire to Institutionalize

Persons with Dementia

\section{References}

1 Van Rensbergen G, Nawrot T. Medical conditions of nursing home admissions. BMC Geriatr. 2010 Jul;10(1): 46.

2 Vandervoort A, Van den Block L, van der Steen JT, Volicer L, Vander Stichele R, Houttekier D, et al. Nursing home residents dying with dementia in Flanders, Belgium: a nationwide postmortem study on clinical characteristics and quality of dying. J Am Med Dir Assoc. 2013 Jul;14(7):485-92.

3 Luppa M, Luck T, Brähler E, König HH, Riedel-Heller SG. Prediction of institutionalisation in dementia. A systematic review. Dement Geriatr Cogn Disord. 2008;26(1):65-78.

4 Gallagher D, Ni Mhaolain A, Crosby L, Ryan D, Lacey L, Coen RF, et al. Determinants of the desire to institutionalize in Alzheimer's caregivers. Am J Alzheimers Dis Other Demen. 2011 May;26(3):205-11.

5 Spitznagel MB, Tremont G, Davis JD, Foster SM. Psychosocial predictors of dementia caregiver desire to institutionalize: caregiver, care recipient, and family relationship factors. J Geriatr Psychiatry Neurol. 2006 Mar; 19(1):16-20.

6 Morycz RK. Caregiving strain and the desire to institutionalize family members with Alzheimer's disease. Possible predictors and model development. Res Aging. 1985 Sep;7(3):329-61.

7 McCaskill GM, Burgio LD, Decoster J, Roff LL. The use of Morycz's desire-to-institutionalize scale across three racial/ethnic groups. J Aging Health. 2011 Feb;23(1):195-202.

8 Spruytte N, Van Audenhove C, Lammertyn F. Predictors of institutionalization of cognitively-impaired elderly cared for by their relatives. Int J Geriatr Psychiatry. 2001 Dec;16(12):1119-28.

9 Vandepitte S, Van Den Noortgate N, Putman K, Verhaeghe S, Faes K, Annemans L. Effectiveness of Supporting Informal Caregivers of People with Dementia: A Systematic Review of Randomized and Non-Randomized Controlled Trials. J Alzheimers Dis. 2016 Apr;52(3):929-65.

10 Barca ML, Engedal K, Laks J, Selbæk G. Quality of life among elderly patients with dementia in institutions. Dement Geriatr Cogn Disord. 2011;31(6):435-42.

11 Gaugler JE, Yu F, Krichbaum K, Wyman JF. Predictors of nursing home admission for persons with dementia. Med Care. 2009 Feb;47(2):191-8.

12 Eska K, Graessel E, Donath C, Schwarzkopf L, Lauterberg J, Holle R. Predictors of institutionalization of dementia patients in mild and moderate stages: a 4-year prospective analysis. Dement Geriatr Cogn Disord Extra. 2013 Nov;3(1):426-45.

13 Gaugler JE, Kane RL, Kane RA, Clay T, Newcomer R. Caregiving and institutionalization of cognitively impaired older people: utilizing dynamic predictors of change. Gerontologist. 2003 Apr;43(2):219-29.

14 López J, Losada A, Romero-Moreno R, Márquez-González M, Martínez-Martín P. [Factors associated with dementia caregivers' preference for institutional care]. Neurologia. 2012 Mar;27(2):83-9.

15 Colucci L, Molino I, Amenta F, Gaeta GL. Desire to institutionalize in Alzheimer's caregivers: an empirical analysis on Italian data. Arch Gerontol Geriatr. 2018 Mar - Apr;75:165-70.

16 Vandepitte S, Van Den Noortgate N, Putman K, Verhaeghe S, Annemans L. Effectiveness and cost-effectiveness of an in-home respite care program in supporting informal caregivers of people with dementia: design of a comparative study. BMC Geriatr. 2016 Dec;16(1):207.

17 Pruchno RA, Michaels JE, Potashnik SL. Predictors of institutionalization among Alzheimer disease victims with caregiving spouses. J Gerontol. 1990 Nov;45(6):S259-66.

18 Zarit SH, Reever KE, Bach-Peterson J. Relatives of the impaired elderly: correlates of feelings of burden. Gerontologist. 1980 Dec;20(6):649-55.

19 Herdman M, Gudex C, Lloyd A, Janssen M, Kind P, Parkin D, et al. Development and preliminary testing of the new five-level version of EQ-5D (EQ-5D-5L). Qual Life Res. 2011 Dec;20(10):1727-36.

20 Devlin NJ, Shah KK, Feng Y, Mulhern B, van Hout B. Valuing health-related quality of life: an EQ-5D-5L value set for England. Health Econ. 2018 Jan;27(1):7-22.

21 Wimo A, Jonsson L, Zbrozek A. The Resource Utilization in Dementia (RUD) instrument is valid for assessing informal care time in community-living patients with dementia. J Nutr Health Aging. 2010 Oct;14(8):685-90.

22 Gustavsson A, Cattelin F, Jönsson L. Costs of care in a mild-to-moderate Alzheimer clinical trial sample: key resources and their determinants. Alzheimers Dement. 2011 Jul;7(4):466-73.

23 Reisberg B, Ferris SH, de Leon MJ, Crook T. The Global Deterioration Scale for assessment of primary degenerative dementia. Am J Psychiatry. 1982 Sep;139(9):1136-9.

24 Katz S, Ford AB, Moskowitz RW, Jackson BA, Jaffe MW. STUDIES OF ILLNESS IN THE AGED. THE INDEX OF ADL: A STANDARDIZED MEASURE OF BIOLOGICAL AND PSYCHOSOCIAL FUNCTION. JAMA. 1963 Sep; 185(12):914-9.

25 Teri L, Truax P, Logsdon R, Uomoto J, Zarit S, Vitaliano PP. Assessment of behavioral problems in dementia: the revised memory and behavior problems checklist. Psychol Aging. 1992 Dec;7(4):622-31.

26 UNESCO. International Standard Classification of Education (ISCED). UNESCO Institute of Statistics; 2018.

27 Bursac Z, Gauss CH, Williams DK, Hosmer DW. Purposeful selection of variables in logistic regression. Source Code Biol Med. 2008 Dec;3(1):17.

28 Zhang Z. Model building strategy for logistic regression: purposeful selection. Ann Transl Med. 2016 Mar; 4(6): 111.

29 Multiple regressieanalyse: associatiemodellen en predictiemodellen. In: Twisk JWR, editor. Inleiding in de toegepaste biostatistiek. Amsterdam, Reed Business Education, 2014; p. 229-274. 
30 Vittinghoff E, McCulloch CE. Relaxing the rule of ten events per variable in logistic and Cox regression. Am J Epidemiol. 2007 Mar;165(6):710-8.

31 Bronselaer J, Vandezande V, Vanden Boer L, Demeyer B. Sporen naar duurzame mantelzorg: hoe perspectief bieden aan mantelzorgers? In: Departement Welzijn VG, editor. Brussels: Flemish Government; 2016.

32 Belle SH, Burgio L, Burns R, Coon D, Czaja SJ, Gallagher-Thompson D, et al.; Resources for Enhancing Alzheimer's Caregiver Health (REACH) II Investigators. Enhancing the quality of life of dementia caregivers from different ethnic or racial groups: a randomized, controlled trial. Ann Intern Med. 2006 Nov; 145(10):727-38.

33 Schulz R, Beach SR. Caregiving as a risk factor for mortality: the Caregiver Health Effects Study. JAMA. 1999 Dec;282(23):2215-9.

34 Sörensen S, Duberstein P, Gill D, Pinquart M. Dementia care: mental health effects, intervention strategies, and clinical implications. Lancet Neurol. 2006 Nov;5(11):961-73.

35 Hébert R, Dubois MF, Wolfson C, Chambers L, Cohen C. Factors associated with long-term institutionalization of older people with dementia: data from the Canadian Study of Health and Aging. J Gerontol A Biol Sci Med Sci. 2001 Nov;56(11):M693-9.

36 Olazarán J, Reisberg B, Clare L, Cruz I, Peña-Casanova J, Del Ser T, et al. Nonpharmacological therapies in Alzheimer's disease: a systematic review of efficacy. Dement Geriatr Cogn Disord. 2010;30(2):161-78.

37 Hurt C, Bhattacharyya S, Burns A, Camus V, Liperoti R, Marriott A, et al. Patient and caregiver perspectives of quality of life in dementia. An investigation of the relationship to behavioural and psychological symptoms in dementia. Dement Geriatr Cogn Disord. 2008;26(2):138-46.

38 Vandepitte S, Van Den Noortgate N, Putman K, Verhaeghe S, Verdonck C, Annemans L. Effectiveness of respite care in supporting informal caregivers of persons with dementia: a systematic review. Int J Geriatr Psychiatry. 2016 Dec;31(12):1277-88.

39 Gaugler JE, Kane RL, Kane RA, Newcomer R. Unmet care needs and key outcomes in dementia. J Am Geriatr Soc. 2005 Dec;53(12):2098-105.

40 Coehlo DP, Hooker K, Bowman S. Institutional placement of persons with dementia: what predicts occurrence and timing? J Fam Nurs. 2007 May;13(2):253-77. 